\title{
Fluid vacation model with Markov modulated load and exhaustive discipline
}

\author{
Zsolt Saffer ${ }^{1}$ and Miklós Telek ${ }^{1,2}$ \\ ${ }^{1}$ Budapest University of Technology and Economics, Hungary \\ ${ }^{2}$ MTA-BME Information systems research group, Hungary \\ \{saffer, telek\}@webspn.hit.bme.hu
}

\begin{abstract}
In this paper we analyze a fluid vacation model with exhaustive discipline, in which the fluid source is modulated by a background continuous-time Markov chain and the fluid is removed by constant rate during the service period. Due to the continuous nature of the fluid the state space of the model becomes continuous, which is the major novelty and challenge of the analysis. We adapt the descendant set approach used in polling models to the fluid vacation model. We provide steady-state vector Laplace Transform and mean of the fluid level at arbitrary epoch.
\end{abstract}

\section{Introduction}

Fluid vacation model is an extension of the classical vacation model (see in [2], [7]), in which fluid takes the role of the customer of the classical model. Due to the the continuous nature of the fluid, the flow in and the removal of fluid are characterized by rates. Hence the state space becomes continuous, which is a challenge in the analysis comparing to that of the discrete state space of the classical vacation model. This requires different analysis techniques.

In this paper we investigate a fluid vacation model with exhaustive service when the fluid source is modulated by a background Markov chain. The main idea of the analysis is the extension of the descendant set approach (see in [1]) to the continuous fluid model context. This together with the transient analysis of the input fluid flow enable to describe the evolution of the joint fluid level and the state of the background Markov chain between the vacation end and vacation start epochs - on the Laplace transform (LT) level. The resulted relations are called as governing equations. From them we determine the steadystate probability vector of the background Markov chain at the vacation start epochs. In the course of the analysis we derive a relation for the steady-state vector LT and vector mean of the fluid level at arbitrary epoch in terms of the previously mentioned steady-state probability vector. We also derive the steadystate LT of the service time, which is the counterpart of the busy period analysis in the classical vacation queue.

The rest of the paper is organized as follows. In section 2 we present the fluid vacation model and the concept of embedding matrix LTs, which is needed to the extension of the descendant set approach to fluid model. In section 3 we 
establish the governing equations of the model. The derivation of steady-state results follows in section 4 . A section with numerical example is eliminated here by space limitation.

\section{Model and Notation}

\subsection{Model description}

We consider a fluid vacation model with Markov modulated load and exhaustive discipline. The model has an infinite fluid buffer.

The input fluid flow of the buffer is determined by a modulating CTMC $(\Omega(t)$ for $t \geq 0)$ with state space $\mathcal{S}=\{1, \ldots, L\}$ and generator $\mathbf{Q}$. When this Markov chain is in state $j(\Omega(t)=j)$ then fluid flows to the buffer at rate $r_{j}$ for $j \in\{1, \ldots, L\}$. We define the diagonal matrix $\mathbf{R}=\operatorname{diag}\left(r_{1}, \ldots, r_{L}\right)$. During the service period the server removes fluid from the fluid buffer at finite rate $d>0$. Consequently, when the overall Markov chain is in state $j(\Omega(t)=j)$ then the fluid level of the buffer during the service period changes at rate $r_{j}-d$, otherwise during the vacation periods it changes at rate $r_{j}$, because there is no service. In the vacation model the length of the service period is determined by the applied discipline. In this work we consider the exhaustive discipline. Under exhaustive discipline the fluid is removed during the service period until the buffer becomes empty. Each time the buffer becomes empty the server takes a vacation period. During vacation periods there is no service thus the fluid level of the buffer is increasing by the actual flowing rates. The consecutive vacation times are independent and identically distributed (i.i.d.). The random variable of the vacation time, its probability distribution function (pdf), its Laplace transform (LT) and its mean are denoted by $\widetilde{\sigma}, \sigma(t)=\frac{d}{d t} \operatorname{Pr}(\widetilde{\sigma}<t)$ and $\sigma^{*}(s)=E\left(e^{-s \widetilde{\sigma}}\right), \sigma=E(\widetilde{\sigma})$, respectively. We define the cycle time (or simple cycle) as the time between just after the starts of two consecutive service periods.

We set the following assumptions on the fluid vacation model:

- A.1 The generator matrix $\mathbf{Q}$ of the modulating CTMC is irreducible.

- A.2 The fluid rates are positive and finite, i.e. $r_{j}>0$ for $j \in\{1, \ldots, L\}$.

- A.3 The fluid is removed from the buffer according to the FCFS discipline.

- A.4 The fluid level decreases during the service period, i.e., $r_{j}<d$ for $j \in\{1, \ldots, L\}$.

Let $\boldsymbol{\pi}$ be the stationary probability vector of the modulating Markov chain. Due to assumption A.1 the equations

$$
\pi \mathbf{Q}=0, \quad \pi \mathbf{e}=1 .
$$

uniquely determine $\boldsymbol{\pi}$, where $\mathbf{e}$ is the $L \times 1$ column vector of ones. The stationary fluid flow rate, $\lambda$, and and the utilization $\rho$, is given as

$$
\lambda=\boldsymbol{\pi} \mathbf{R e}, \quad \rho=\frac{\lambda}{d},
$$


respectively. The necessary condition of the stability of the fluid vacation model is that mean fluid arrival rate $\lambda=\boldsymbol{\pi} \mathbf{R e}$ is less than $d$, which is equivalent with $\rho<1$.

If the amount of fluid served during a service period were limited, like e.g. in case of a model with time-limited discipline, then further restriction would be needed for the sufficiency. However the model with the exhaustive discipline does not have any load-independent limitation for a service period, therefore the above necessary condition is also a sufficient one for the stability of the system.

For the $i, j$-th element of the matrix $\mathbf{Z}$ the notations $\mathbf{Z}_{i j}$ or $[\mathbf{Z}]_{i j}$ are used. Similarly $\mathbf{z}_{j}$ and $[\mathbf{z}]_{j}$ denote the $j$-th element of vector $\mathbf{z}$. When $\mathbf{X}^{*}(s), \operatorname{Re}(s) \geq 0$ is a matrix LT, $\mathbf{X}^{(k)}$ denotes its $k$-th $(k \geq 1)$ derivative at $s=0$, i.e., $\mathbf{X}^{(k)}=$ $\left.\frac{d^{k}}{d s^{k}} \mathbf{X}^{*}(s)\right|_{s=0}$ and $\mathbf{X}$ denotes its value at $s=0$, i.e., $\mathbf{X}=\mathbf{X}^{*}(0)$. Similar notations are applied for vector $\mathrm{LT} \mathbf{x}^{*}(s)$ and scalar LT $x^{*}(s)$.

\subsection{Embedded matrix LTs}

Let $\mathbf{Z}$ be an $L \times L$ rate matrix which has the following properties:

- the diagonal elements are negative $\left(\mathbf{Z}_{i, i}<0\right)$ and the other elements are non-negative $\left(\mathbf{Z}_{i, j} \geq 0\right.$, for $\left.i \neq j\right)$,

- the row sums are zero.

For $\operatorname{Re}(v) \geq 0$ let

$$
\mathbf{H}(v)=\mathbf{T} v-\mathbf{Z},
$$

be a linear $L \times L$ matrix function of the complex variable $v$, where $\mathbf{Z}$ is a rate matrix and $\mathbf{T}$ is diagonal and its diagonal elements are positive, i.e. $[\mathbf{T}]_{j, j}>0$ for $j \in\{1, \ldots, L\}$. That is $\mathbf{Z}$ and $\mathbf{T}$ are real. The matrix function $-\mathbf{H}(v)$ has the following properties:

- P.1 it is analytic for $\operatorname{Re}(v) \geq 0$,

- P.2 it is a rate matrix when $v=0$,

- P.3 the real part of its diagonal elements are negative for $\operatorname{Re}(v) \geq 0$, i.e. $\left(\operatorname{Re}\left(-\mathbf{H}_{j, j}(v)\right)<0\right)$,

- P.4 it is a diagonal dominant matrix for $\operatorname{Re}(v) \geq 0$, i.e., $\left|\operatorname{Re}\left(-\mathbf{H}_{j, j}(v)\right)\right| \geq$ $\left.\sum_{k, k \neq j} \mid-\mathbf{H}_{j, k}(v)\right) \mid$.

We define the operator $\mathcal{O}()$ on a complex variable $v$ and on a linear matrix function $\mathbf{G}(v)=\mathbf{G}_{\mathbf{1}} v+\mathbf{G}_{\mathbf{2}}$ as the operator performing the substitution $v \rightarrow$ $\mathbf{H}(v)$. That is $\mathcal{O}(v)=\mathbf{H}(v)=\mathbf{T} v-\mathbf{Z}$ and $\mathcal{O}(\mathbf{G}(v))=\mathbf{G}_{\mathbf{1}} \mathbf{H}(v)+\mathbf{G}_{\mathbf{2}}=\mathbf{G}_{\mathbf{1}} \mathbf{T} v-$ $\mathbf{G}_{\mathbf{1}} \mathbf{Z}+\mathbf{G}_{\mathbf{2}}$, which are linear matrix functions as well. The order of non-commuting matrices are kept according to this definition. The multifold operator $\mathcal{O}^{k}(\bullet)$ is defined recursively as $\mathcal{O}^{k}(\bullet)=\mathcal{O}\left(\mathcal{O}^{k-1}(\bullet)\right), \quad k \geq 2$, where $\mathcal{O}^{1}(\bullet)=\mathcal{O}(\bullet)$ by definition. Additionally, we introduce $\mathcal{O}^{0}(\bullet)=\bullet$. Starting from (3), the $L \times L$ linear matrix function $\mathcal{O}^{k}(v)$ can be expressed recursively as

$$
\mathcal{O}^{k}(v)=\mathbf{T}^{k} v-\sum_{i=0}^{k-1} \mathbf{T}^{i} \mathbf{Z}
$$


The matrix function $-\mathcal{O}^{k}(v)$ has the following properties:

$\diamond-\mathcal{O}^{k}(v)$ is analytic for $\operatorname{Re}(v) \geq 0$ (due to $\mathbf{P . 1}$ of $\mathbf{H}(v)$ ),

$\diamond-\left.\mathcal{O}^{k}(v)\right|_{v=0}$ is also a rate matrix (P.2), since multiplying rate matrix $\mathbf{Z}$ any times by positive diagonal matrices from left results in a rate matrix and the sum of $L \times L$ rate matrices is also an $L \times L$ rate matrix,

$\diamond-\mathcal{O}^{k}(v)$ has also the properties P.3 and P.4.

It follows from the recursive definition of the multifold operator $\mathcal{O}^{k}()$ that the matrix $\operatorname{LT} \int_{x=0}^{\infty} p(x) e^{-\mathcal{O}^{k}(v) x} d x$ is created by consecutive embedding of the matrix $\mathbf{H}(v)$ in the previous matrix LT and therefore we call this matrix LT as embedded matrix LT. If $p(x) \geq 0$ for $x \geq 0$ and $v$ is the complex argument of the LT $\int_{x=0}^{\infty} p(x) e^{-v x} d x$ then

$$
\int_{x=0}^{\infty} p(x) e^{-\mathcal{O}(v) x} d x=\int_{x=0}^{\infty} p(x) e^{-\mathbf{H}(v) x} d x
$$

is an $L \times L$ matrix LT. According to the Gerschgorin Circle Theorem [3] each eigenvalue of $-\mathbf{H}(v)$ is in one of the disks $\left\{z:\left|z-\left(-\mathbf{H}_{j, j}(v)\right)\right| \leq \sum_{k \neq j} \mid-\right.$ $\left.\mathbf{H}_{j, k}(v) \mid\right\}$ (i.e. disks in complex z-plane with center at $\left(-\mathbf{H}_{j, j}(v)\right)$ and radius $\left.\sum_{k \neq j}\left|-\mathbf{H}_{j, k}(v)\right|\right)$, for $\forall j \in\{1, \ldots, L\}$. This together with properties $\mathbf{P . 3}$ and $\mathbf{P . 4}$ imply that the eigenvalues of $-\mathbf{H}(v)$ have negative or zero real part for $\operatorname{Re}(v) \geq 0$. The matrix function $e^{-\mathbf{H}(v) x}$ can be written in Lagrange matrix polynomial form as

$$
\left(e^{x}\right)^{-\mathbf{H}(v)}=\sum_{k=1}^{K}\left(e^{x}\right)^{\gamma_{k}} L_{k}(-\mathbf{H}(v))
$$

where $K$ is the number of different eigenvalues of matrix $(-\mathbf{H}(v)), \gamma_{k}$ and $L_{k}(v)$, for $k=1, \ldots, K$ denotes the different eigenvalues of matrix $(-\mathbf{H}(v))$ and the finite Lagrange-polynomials belonging to the roots of the minimal-polynomial of matrix $(-\mathbf{H}(v))$, respectively. Applying (6) in (5) and rearranging it yields

$$
\int_{x=0}^{\infty} p(x) e^{-\mathcal{O}(v) x} d x=\sum_{k=1}^{K} L_{k}(-\mathbf{H}(v)) \int_{x=0}^{\infty} p(x) e^{\gamma_{k} x} d x
$$

Recall that the eigenvalues $\gamma_{k}$ have negative or zero real part for $\operatorname{Re}(v) \geq 0$. Consequently (5) is finite when the $\operatorname{LT} \int_{x=0}^{\infty} p(x) e^{-v x} d x$ is finite for $\operatorname{Re}(v) \geq 0$.

The same argument holds also for the matrix LT $\int_{x=0}^{\infty} p(x) e^{-\mathcal{O}^{k}(v) x} d x$, since the utilized properties P.3 and P.4 of $(-\mathbf{H}(v))$ hold also for $-\mathcal{O}^{k}(v)$. We remark here that the order of matrix and scalar $\mathbf{T} v$ in the definition of $\mathbf{H}(v)$ is crucial in order to ensure the validity of the properties $\mathbf{P . 2}$ and $\mathbf{P . 4}$ for the matrix function $\mathcal{O}^{k}(v)$.

\section{The governing equations of the system}

\subsection{Transient analysis of the arriving fluid}

In this section we consider the accumulated fluid during time $t \geq 0$. More precisely we derive the matrix LT of the fluid flowing into the buffer as a function 
of time, where the rows and columns of the matrix LT represent the initial and the final states of the modulating Markov chain.

Let $Y(t) \in \mathbb{R}^{+}$be the accumulated fluid arrived at the buffer until time $t, \mathbf{A}(t, y)$ be the transition density matrix composed by elements $\mathbf{A}_{j, k}(t, y)=$ $\frac{\partial}{\partial y} \operatorname{Pr}(\Omega(t)=k, Y(t)<y \mid \Omega(0)=j, Y(0)=0)$ and its Laplace transform be $\mathbf{A}^{*}(t, v)=\int_{y=0}^{\infty} \mathbf{A}(t, y) e^{-v y} d y$.

Proposition 1. The matrix LT of the fluid generated by the Markov modulated fluid source in interval $(0, t]$ can be expressed as

$$
\mathbf{A}^{*}(t, v)=e^{-t(\mathbf{R} v-\mathbf{Q})} .
$$

The proof of the proposition is provided in [6].

\subsection{The descendant fluid}

We extend the of descendant set (see in Borst and Boxma [1]) to fluid model and describe the exhaustive service period as consecutive gated service intervals without vacations. We define the 1-st descendant fluid level of the given fluid amount as the fluid flowing into the buffer during the service of the given fluid amount. This is similar to the descendant set of a customer in the regular vacation model, which consists of the group of customers arrived during the service of the original customer. Similarly we define the $k$-th descendant fluid level of the given fluid amount recursively as the fluid accumulated during the service of the $k-1$-th descendant fluid level. This is the same as the fluid level after $k$ cycles in a gated system without vacation initiated by the given fluid amount. By definition the 0-th descendant fluid level of a given fluid amount equals to itself. The $k$-th descendant period is defined as the removal time of the $k$-th descendant fluid for $k \geq 0$. Moreover we consider the evolution of the fluid level jointly with the evolution of the state of the modulating Markov chain. This joint evolution is described by the help of matrix LT formalism. When $\mathbf{g}^{*}(v)$ is the vector LT of a given initial fluid density then the pdf and the vector LT of its $k$-th descendant fluid level, for $k \geq 1$, are denoted by $\mathbf{g}^{<k>}(x)$ and $\mathbf{g}^{*<k>}(v)$. Furthermore $\mathbf{g}^{<0>}(x)=\mathbf{g}(x)$ and $\mathbf{g}^{*<0>}(v)=\mathbf{g}^{*}(v)$. Let

$$
\mathcal{O}(v)=\mathbf{H}(v)=\frac{\mathbf{R} v-\mathbf{Q}}{d},
$$

that is $\mathbf{T}=\frac{\mathbf{R}}{d}$ and $\mathbf{Z}=\frac{\mathbf{Q}}{d}$ in (3).

Furthermore we introduce a notation for the LT with respect to the $L \times L$ matrix function $\mathbf{H}(v)$ as follows

$$
\mathbf{g}^{*}(\mathbf{H}(v))=\int_{x=0}^{\infty} \mathbf{g}(x) e^{-\mathbf{H}(v) x} d x,
$$

where $\mathbf{g}()$ is an $1 \times L$ vector function. 
Proposition 2. Starting from the initial fluid amount whose vector $L T$ is $\mathbf{g}^{*}(v)$ the vector $L T$ of the $k$-th $(k \geq 0)$ descendant fluid can be expressed as

$$
\mathbf{g}^{*<k>}(v)=\mathbf{g}^{*}\left(\mathcal{O}^{k}(v)\right) .
$$

Proof. The $k$-th descendant fluid is defined as the fluid accumulated during the service of the $k-1$-th descendant fluid for $k \geq 1$. The fluid density vector of the $k-1$-th descendant fluid is $\mathbf{g}^{<k-1>}(\xi)$. When the $k-1$-th descendant fluid is $\xi$, then its service duration is $\frac{\xi}{d}$, from which we can express $\left[\mathbf{g}^{<k>}(x)\right]_{k}$ as

$$
\left[\mathbf{g}^{<k>}(x)\right]_{k}=\sum_{j=1}^{L} \int_{\xi=0}^{\infty}\left[\mathbf{g}^{<k-1>}(\xi)\right]_{j} \mathbf{A}_{j k}\left(\frac{\xi}{d}, x\right) d \xi,
$$

whose vector-matrix form is

$$
\mathbf{g}^{<k>}(x)=\int_{\xi=0}^{\infty} \mathbf{g}^{<k-1>}(\xi) \mathbf{A}\left(\frac{\xi}{d}, x\right) d \xi .
$$

Applying (7) the LT of $\mathbf{g}^{<k>}(x)$ with respect to $x$ is

$$
\mathbf{g}^{*<k>}(v)=\int_{\xi=0}^{\infty} \mathbf{g}^{<k-1>}(\xi) \mathbf{A}^{*}\left(\frac{\xi}{d}, v\right) d \xi=\int_{\xi=0}^{\infty} \mathbf{g}^{<k-1>}(\xi) e^{-\frac{\xi}{d}(\mathbf{R} v-\mathbf{Q})} d \xi .
$$

Utilizing that the right hand side of (12) is a matrix LT according to (9) and using (8) we have

$$
\mathbf{g}^{*<k>}(v)=\mathbf{g}^{*<k-1>}\left(\frac{\mathbf{R} v-\mathbf{Q}}{d}\right)=\mathbf{g}^{*<k-1>}(\mathbf{H}(v))=\mathbf{g}^{*<k-1>}(\mathcal{O}(v)) .
$$

Using the definition $\mathbf{g}^{*<0>}(v)=\mathbf{g}^{*}(v)$ for $k=1$ we get

$$
\mathbf{g}^{*<1>}(v)=\mathbf{g}^{*}(\mathcal{O}(v)) \text {. }
$$

Applying (14) recursively in (13) gives the proposition for $k \geq 2$. For $k=0$ the proposition follows from the definitions $\mathbf{g}^{*<0>}(v)=\mathbf{g}^{*}(v)$ and $\mathcal{O}^{0}(v)=v$.

Proposition 3. If the diagonal elements of $\frac{\mathbf{R}}{d}$ are less than one then $\lim _{k \rightarrow \infty} \mathcal{O}^{k}(v)$ exists, finite, independent of $v$ and it is

$$
\mathcal{O}^{\infty}(v)=\lim _{k \rightarrow \infty} \mathcal{O}^{k}(v)=\left(\frac{\mathbf{R}}{d}-\mathbf{I}\right)^{-1} \frac{\mathbf{Q}}{d} .
$$

Proof. Applying $\mathbf{T}=\frac{\mathbf{R}}{d}$ and $\mathbf{Z}=\frac{\mathbf{Q}}{d}$ in (4) gives

$$
\mathcal{O}^{k}(v)=\left(\frac{\mathbf{R}}{d}\right)^{k} v-\sum_{i=0}^{k-1}\left(\frac{\mathbf{R}}{d}\right)^{i} \frac{\mathbf{Q}}{d} .
$$

When the diagonal elements of $\frac{\mathbf{R}}{d}$ are less than one then $\lim _{k \rightarrow \infty}\left(\frac{\mathbf{R}}{d}\right)^{k}=\mathbf{0}$ and $\lim _{k \rightarrow \infty} \sum_{i=0}^{k-1}\left(\frac{\mathbf{R}}{d}\right)^{i}=\left(\mathbf{I}-\frac{\mathbf{R}}{d}\right)^{-1}$. Applying them in (16) results in the proposition. 
Proposition 4. Starting from the initial fluid amount whose vector $L T$ is $\mathbf{g}^{*}(v)$ the limiting vector $L T$ of the $k$-th descendant fluid as $k$ tends to infinity can be expressed as

$$
\lim _{k \rightarrow \infty} \mathbf{g}^{*<k>}(v)=\mathbf{g}^{*}\left(\mathcal{O}^{\infty}(v)\right)=\mathbf{g}^{*}\left(\left(\frac{\mathbf{R}}{d}-\mathbf{I}\right)^{-1} \frac{\mathbf{Q}}{d}\right) .
$$

Proof. Applying (10) and the operator limit (15) we have

$$
\lim _{k \rightarrow \infty} \mathbf{g}^{*<k>}(v)=\mathbf{g}^{*}\left(\lim _{k \rightarrow \infty} \mathcal{O}^{k}(v)\right)=\mathbf{g}^{*}\left(\mathcal{O}^{\infty}(v)\right)=\mathbf{g}^{*}\left(\left(\frac{\mathbf{R}}{d}-\mathbf{I}\right)^{-1} \frac{\mathbf{Q}}{d}\right) .
$$

\subsection{The governing equations of the system at vacation start and end epochs}

Let $X(t) \in \mathbb{R}^{+}$denote the fluid level in the buffer at time $t$ and $t^{f}(\ell)$ for $\ell \geq 1$ be the time at the end of the vacation in the $\ell$-th cycle. We define the $1 \times L$ row vector $\mathbf{f}(\ell, x)$ by its elements as

$$
[\mathbf{f}(\ell, x)]_{j}=\frac{d}{d x} \operatorname{Pr}\left(\Omega\left(t^{f}(\ell)\right)=j, X\left(t^{f}(\ell)\right)<x\right), j \in \Omega,
$$

and its $\operatorname{LT}$ as $\mathbf{f}^{*}(\ell, v)=\int_{x=0}^{\infty} \mathbf{f}(\ell, x) e^{-v x} d x$. We also define the steady-state vector LT of the fluid level at end of vacation, the $1 \times L$ row vector $\mathbf{f}^{*}(v)$ as $\mathbf{f}^{*}(v)=$ $\lim _{\ell \rightarrow \infty} \mathbf{f}^{*}(\ell, v)$. Analogously let $t^{m}(\ell)$ be the time at the start of vacation in the $\ell$-th cycle. The $1 \times L$ row vector $\mathbf{m}(\ell, x)$ is defined by its elements as

$$
[\mathbf{m}(\ell, x)]_{j}=\frac{d}{d x} \operatorname{Pr}\left(\Omega\left(t^{m}(\ell)\right)=j, X\left(t^{m}(\ell)\right)<x\right), j \in \Omega,
$$

and its LT and embedded steady-state vector are $\mathbf{m}^{*}(\ell, v)=\int_{x=0}^{\infty} \mathbf{m}(\ell, x) e^{-v x} d x$ and $\mathbf{m}^{*}(v)=\lim _{\ell \rightarrow \infty} \mathbf{m}^{*}(\ell, v)$.

Due to the exhaustive discipline the buffer is idle at the start of the vacation period. This implies that $\mathbf{m}^{*}(\ell, v)$ is independent of $v$, which is the phase distribution of the background Markov chain at the beginning of the $\ell$ th vacation. Thus we introduce $\mathbf{m}(\ell)=\mathbf{m}^{*}(\ell, v)$.

Theorem 1. In the fluid vacation model with exhaustive discipline the vector LTs of the fluid level at the end of the $\ell$ th vacation, $\mathbf{f}^{*}(\ell, v), \ell \geq 0$, and at the start of the $\ell$ th vacation, $\mathbf{m}(\ell), \ell \geq 1$, satisfy

$$
\begin{gathered}
\mathbf{m}(\ell)=\mathbf{f}^{*}\left(\ell-1, \mathcal{O}^{\infty}(v)\right)=\mathbf{f}^{*}\left(\ell-1,\left(\frac{\mathbf{R}}{d}-\mathbf{I}\right)^{-1} \frac{\mathbf{Q}}{d}\right), \\
\mathbf{f}^{*}(\ell, v)=\mathbf{m}(\ell) \sigma^{*}(\mathbf{R} v-\mathbf{Q}) .
\end{gathered}
$$


Proof. The $k$-th descendant fluid as $\lim _{k \rightarrow \infty}$ gives the fluid at the end of the exhaustive service period. Hence starting a service period with initial joint fluid level and phase distribution $\mathbf{g}^{*}(v)$ at the end of the service period the joint fluid level and phase distribution is $\lim _{k \rightarrow \infty} \mathbf{g}^{*<k>}(v)$. Proposition 4 states that $\mathbf{g}^{*}\left(\mathcal{O}^{\infty}(v)\right)=\mathbf{g}^{*}\left(\left(\frac{\mathbf{R}}{d}-\mathbf{I}\right)^{-1} \frac{\mathbf{Q}}{d}\right) \cdot(18)$ comes from the fact that the joint fluid level and phase distribution at the end of the $\ell-1$ th vacation period is $\mathbf{f}^{*}(\ell-1, v)$.

In the exhaustive system the fluid level at the end of the $\ell$ th vacation equals the fluid flowed into the buffer during the $\ell$ th vacation, since the buffer is idle at the start of the $\ell$ th vacation. Taking into account also the state of the modulating Markov chain we have

$$
[\mathbf{f}(\ell, x)]_{k}=\sum_{j=1}^{L} \int_{t=0}^{\infty}[\mathbf{m}(\ell)]_{j} \mathbf{A}_{j k}(t, x) \sigma(t) d t
$$

Rearranging (20) to matrix form we get

$$
\mathbf{f}^{*}(\ell, v)=\int_{t=0}^{\infty} \mathbf{m}(\ell) \mathbf{A}^{*}(t, v) \sigma(t) d t=\mathbf{m}(\ell) \int_{t=0}^{\infty} e^{-t(\mathbf{R} v-\mathbf{Q})} \sigma(t) d t
$$

where the explicit form of $\mathbf{A}^{*}(t, v)$ is taken from (7). Rearranging (21) results in (19).

In the rest of the paper we avoid the scalar versions of the equations like (11) and (20) and directly write their matrix versions.

\section{The steady-state behavior of the fluid vacation model}

The main goal of this section is to compute the time stationary distribution of the fluid vector in transform domain. To this end we first provide the stationary distribution in service start and end epochs in Sec. 4.1, collect some subsequently used general properties of fluid vacation models in Sec. 4.2, compute the service time distribution in Sec. 4.3 and finally evaluate the time stationary behavior in Sec. 4.4.

\subsection{Steady-state behavior at start and end of vacation}

We define $\mathbf{m}$ as $\mathbf{m}=\lim _{\ell \rightarrow \infty} \mathbf{m}(\ell)$ and $\mathbf{e}$ as the column vector of ones.

Theorem 2. The stationary behavior of the stable fluid vacation model with exhaustive discipline is characterized by $\mathbf{m}$ and $\mathbf{f}^{*}(v)$ where $\mathbf{m}$ is the solution of the linear system

$$
\begin{aligned}
\mathbf{m} & =\mathbf{m} \sigma^{*}\left(\left(\frac{\mathbf{R}}{d}-\mathbf{I}\right)^{-1} \mathbf{Q}\right), \\
\mathbf{m e} & =1
\end{aligned}
$$


and

$$
\mathbf{f}^{*}(v)=\mathbf{m} \sigma^{*}(\mathbf{R} v-\mathbf{Q}) .
$$

Proof. Applying (19) in (18) gives

$$
\mathbf{m}(\ell)=\mathbf{m}(\ell-1) \sigma^{*}\left(\mathbf{R} \mathcal{O}^{\infty}(v)-\mathbf{Q}\right) .
$$

Taking the limit $\ell \rightarrow \infty$ in (24) and rearranging it leads to

$$
\mathbf{m}=\mathbf{m} \sigma^{*}\left(d \frac{\mathbf{R} \mathcal{O}^{\infty}(v)-\mathbf{Q}}{d}\right)=\mathbf{m} \sigma^{*}\left(d \mathcal{O}\left(\mathcal{O}^{\infty}(v)\right)\right)=\mathbf{m} \sigma^{*}\left(d \mathcal{O}^{\infty}(v)\right) .
$$

Applying (15) in (25) results in the first equation of (22). The normalizing condition of the system of linear equations comes from the fact that $\mathbf{m}$ is the phase distribution of the background Markov chain of the fluid source at service completion. Finally (23) comes by taking the limit $\ell \rightarrow \infty$ in (19).

\subsection{Equilibrium relationships}

Let $S(\ell)$ be the service time in the $\ell$-th cycle, $C(\ell)$ be the cycle time between two consecutive service starts in the $\ell$-th cycle, $Z(\ell)$ be the amount of fluid served in the $\ell$-th cycle, and $Z_{i}(\ell)$ be the amount of fluid served in the $i$-th descendant period of the $\ell$-th service period. The related $\lim _{\ell \rightarrow \infty}$ stationary quantities are $S, C, Z$, and $Z_{i}$, their LTs are $s^{*}(v), c^{*}(v), z^{*}(v), z_{i}^{*}(v)$, and their means are $s, c, z, z_{i}$, respectively. The definitions imply that $S d=Z, C=S+\tilde{\sigma}$ and $Z=\sum_{i=0}^{\infty} Z_{i}$ hold as well as these relations hold for their respective means.

Let $Y(t)$ be the accumulated fluid flowed into the buffer in interval $(0, t]$ and $a$ be the mean amount of fluid, which flows into the buffer during a cycle in steady state. That is

$$
a=\lim _{k \rightarrow \infty} \frac{\sum_{\ell=1}^{k} E\left[Y\left(t^{f}(\ell+1)\right)-Y\left(t^{f}(\ell)\right)\right]}{k},
$$

whose right hand side can be rearranged as

$$
a=\lim _{k \rightarrow \infty} \frac{E\left[\sum_{\ell=1}^{k} Y\left(t^{f}(\ell+1)\right)-Y\left(t^{f}(\ell)\right)\right]}{E\left[\sum_{\ell=1}^{k} C(\ell)\right]} \lim _{k \rightarrow \infty} \frac{E\left[\sum_{\ell=1}^{k} C(\ell)\right]}{k}=\lambda c .
$$

Corollary 1. In the stable fluid vacation model the steady-state mean cycle time can be expressed as

$$
c=\frac{\sigma}{1-\rho} .
$$

Proof. In the stable fluid vacation model the amount of fluid flowing into the buffer during a cycle equals the amount of fluid removed during the service period, that is $a=s d$. From this and $a=\lambda c$ we get $s=\frac{\lambda}{d} c=\rho c$ and $c=\sigma+s=$ $\sigma+\rho c$, which gives the statement. 


\subsection{The steady-state distribution of the service time}

Theorem 3. The amount of fluid served in a service period and the length of the service period satisfy

$$
\begin{gathered}
z^{*}(v)=\mathbf{f}^{*}\left(\left(\mathbf{I}-\frac{\mathbf{R}}{d}\right)^{-1}\left(v \mathbf{I}-\frac{\mathbf{Q}}{d}\right)\right) \mathbf{e}, \\
s^{*}(v)=z^{*}(v / d) .
\end{gathered}
$$

Proof. Let $f_{Z_{0}, Z_{1}, \ldots, Z_{k}}\left(x_{0}, x_{1}, \ldots, x_{k}\right)$ denote the joint density of $Z_{0}, Z_{1}, \ldots, Z_{k}$. Furthermore let the matrix $\mathbf{A}\left(\frac{x_{i}}{d}, x_{i+1}\right)$ stand for the state dependent density of the fluid arrived to the buffer during the $\frac{x_{i}}{d}$ long $i$-th descendant period for $i=0, \ldots, k-1$.

The fluid served in the $i$-th descendant period is the fluid flowed into the buffer during the $i-1$-th descendant period for $i=1, \ldots, k$. Using it and taking into account also the evolution of the modulating Markov state the joint density $f_{Z_{0}, Z_{1}, \ldots, Z_{k}}\left(x_{0}, x_{1}, \ldots, x_{k}\right)$ can be given as

$$
f_{Z_{0}, Z_{1}, \ldots, Z_{k}}\left(x_{0}, x_{1}, \ldots, x_{k}\right)=\mathbf{f}\left(x_{0}\right) \mathbf{A}\left(\frac{x_{0}}{d}, x_{1}\right) \ldots \mathbf{A}\left(\frac{x_{k-1}}{d}, x_{k}\right) \mathbf{e} .
$$

By the help of (30) the mean $E\left(e^{-v \sum_{i=0}^{k} Z_{i}}\right)$ can be expressed as

$$
\begin{aligned}
& E\left(e^{-v \sum_{i=0}^{k} Z_{i}}\right)= \\
& \int_{x_{0}} \int_{x_{1}} \ldots \int_{x_{k}} E\left(e^{-v \sum_{i=0}^{k} Z_{i}} \mid Z_{0}=x_{0}, Z_{1}=x_{1}, \ldots, Z_{k}=x_{k}\right) \\
& \times f_{Z_{0}, Z_{1}, \ldots, Z_{k}}\left(x_{0}, x_{1}, \ldots, x_{k}\right) d x_{k} \ldots d x_{1} d x_{0}= \\
& \int_{x_{0}} \mathbf{f}\left(x_{0}\right) \int_{x_{1}} \mathbf{A}\left(\frac{x_{0}}{d}, x_{1}\right) \ldots \underbrace{\int_{x_{k}} \mathbf{A}\left(\frac{x_{k-1}}{d}, x_{k}\right) e^{-v x_{k}} d x_{k}}_{\mathbf{A}^{*}\left(\frac{x_{k-1}}{d}, v\right)} \ldots e^{-v x_{1}} d x_{1} e^{-v x_{0}} d x_{0} \mathbf{e}= \\
& \int_{x_{0}} \mathbf{f}\left(x_{0}\right) \int_{x_{1}} \mathbf{A}\left(\frac{x_{0}}{d}, x_{1}\right) \ldots \underbrace{\int_{x_{k-1}} \mathbf{A}\left(\frac{x_{k-2}}{d}, x_{k-1}\right) \underbrace{e^{-x_{k-1} \frac{\mathbf{R} v-\mathbf{Q}}{d} e^{-v x_{k-1}}} d x_{k-1}}_{e^{-x_{k-1}(\mathcal{O}(v)+\mathbf{I} v)}}}_{\mathbf{A}^{*}\left(\frac{x_{k-2}}{d}, \mathcal{O}(v)+\mathbf{I} v\right)} \\
& \ldots e^{-v x_{1}} d x_{1} e^{-v x_{0}} d x_{0} \mathbf{e}= \\
& \int_{x_{0}} \mathbf{f}\left(x_{0}\right) \int_{x_{1}} \mathbf{A}\left(\frac{x_{0}}{d}, x_{1}\right) \ldots \underbrace{\int_{x_{k-2}} \mathbf{A}\left(\frac{x_{k-3}}{d}, x_{k-2}\right) e^{-x_{k-2} \frac{\mathbf{R}(\mathcal{O}(v)+\mathbf{I} v)-\mathbf{Q}}{d} e^{-v x_{k-2}} d x_{k-2}}}_{\mathbf{A}^{*}\left(\frac{x_{k-3}}{d}, \mathcal{O}^{2}(v)+\frac{\mathbf{R}}{d} v+\mathbf{I} v\right)} \\
& \ldots e^{-v x_{1}} d x_{1} e^{-v x_{0}} d x_{0} \mathbf{e}= \\
& \ldots=\mathbf{f}^{*}\left(\mathcal{O}^{k}(v)+\sum_{i=0}^{k-1}\left(\frac{\mathbf{R}}{d}\right)^{i} v\right) \mathbf{e} .
\end{aligned}
$$


This together with (15) yields

$$
\begin{aligned}
z^{*}(v) & =E\left(e^{-v \sum_{i=0}^{\infty} Z_{i}}\right)=\mathbf{f}^{*}\left(\mathcal{O}^{\infty}(v)+\sum_{i=0}^{\infty}\left(\frac{\mathbf{R}}{d}\right)^{i} v\right) \mathbf{e}= \\
& =\mathbf{f}^{*}\left(\left(\frac{\mathbf{R}}{d}-\mathbf{I}\right)^{-1} \frac{\mathbf{Q}}{d}+\left(\mathbf{I}-\frac{\mathbf{R}}{d}\right)^{-1} v\right) \mathbf{e},
\end{aligned}
$$

from which rearrangement results in the first statement of the theorem. For the service time distribution we have

$$
s^{*}(v)=E\left(e^{-v S}\right)=E\left(e^{-v Z / d}\right)=E\left(e^{-(v / d) Z}\right)=z^{*}(v / d) .
$$

\subsection{The steady-state vector LT of the fluid level}

The steady-state joint distribution of the fluid level and the state of the modulating Markov chain at an arbitrary epoch is defined by the $1 \times L$ row vector $\mathbf{q}(x)$ whose $j$-th element is

$$
[\mathbf{q}(x)]_{j}=\lim _{t \rightarrow \infty} \frac{d}{d x} \operatorname{Pr}(\Omega(t)=j, X(t)<x), j \in \Omega .
$$

The LT of $\mathbf{q}(x)$ is $\mathbf{q}^{*}(v)=\int_{x=0}^{\infty} \mathbf{q}(x) e^{-v x} d x$.

Let $d_{k}$ be the start time of the $k$ th descendant service period for $k \geq 0$, where $d_{0}=0$. The steady-state joint density of the fluid level and the state of the modulating Markov chain at an arbitrary epoch in the $k$ th $(k \geq 0)$ descendant service period, the $1 \times L$ row vector $\mathbf{q}_{k}(x)$ is defined by its $j$-th element as

$$
\left[\mathbf{q}_{k}(x)\right]_{j}=\lim _{t \rightarrow \infty} \frac{d}{d x} \operatorname{Pr}\left(\Omega(t)=j, X(t)<x \mid t \in\left(d_{k}, d_{k+1}\right)\right), j \in \Omega .
$$

and corresponding LT is $\mathbf{q}_{k}^{*}(v)$.

Let $1_{(\text {con })}$ denote the indicator of condition "con". Furthermore let $\mathbf{e}_{j}=$ $(0, \ldots, 0,1,0, \ldots, 0)$ be the $1 \times L$ vector with 1 at the $j$-th position. We define the $1 \times L$ indicator vector $\mathbf{1}_{(\Omega(t))}$ as $\mathbf{1}_{(\Omega(t))}=\sum_{j=1}^{L} 1_{(\Omega(t)=j)} \mathbf{e}_{j}$.

Proposition 5. For $k \geq 0$ the LT of the mean fluid level for the $k$ th descendant interval, $E\left[\int_{t=d_{k}}^{d_{k+1}} e^{-X(t) v} \mathbf{1}_{(\Omega(t))} d t\right]$, satisfies

$$
E\left[\int_{t=d_{k}}^{d_{k+1}} e^{-X(t) v} \mathbf{1}_{(\Omega(t))} d t\right]((\mathbf{R}-d \mathbf{I}) v-\mathbf{Q})=\mathbf{f}^{*}\left(\mathcal{O}^{k}(v)\right)-\mathbf{f}^{*}\left(\mathcal{O}^{k+1}(v)\right) .
$$

Proof. If the fluid level at the beginning of the $k$-th descendant period is $x_{k}$ then the fluid level after time $t$ in the $k$-th descendant period is $x_{k}-t d+A(t)$ where $A(t)$ denotes the amount of fluid arrived in $(0, t)$ in the $k$-th descendant period. The LT of this quantity is $E\left(e^{-v\left(x_{k}-t d+A(t)\right)}\right)=E\left(e^{-v A(t)}\right) e^{-v\left(x_{k}-t d\right)}$, where the first term is the LT of $A(t)$. Considering the state dependency of fluid 
level at the beginning of the $k$-th descendant period and the fluid arrival process for $k>0$ we have

$E\left[\int_{t=d_{k}}^{d_{k+1}} e^{-X(t) v} \mathbf{1}_{(\Omega(t))} d t\right]=$ $\int_{x_{0}} \mathbf{f}\left(x_{0}\right) \int_{x_{1}} \mathbf{A}\left(\frac{x_{0}}{d}, x_{1}\right) \ldots \int_{x_{k}} \mathbf{A}\left(\frac{x_{k-1}}{d}, x_{k}\right) \int_{t=0}^{x_{k} / d} \mathbf{A}^{*}(t, v) e^{-\left(x_{k}-t d\right) v} d t d x_{k} \ldots d x_{0}=$ $\int_{x_{0}} \mathbf{f}\left(x_{0}\right) \int_{x_{1}} \mathbf{A}\left(\frac{x_{0}}{d}, x_{1}\right) \ldots \int_{x_{k}} \mathbf{A}\left(\frac{x_{k-1}}{d}, x_{k}\right) \underbrace{\int_{t=0}^{x_{k} / d} e^{-t((\mathbf{R}-d \mathbf{I}) v-\mathbf{Q})} d t} e^{-x_{k} v} d x_{k} \ldots d x_{0}$.

The underbraced integral can be evaluated by means of the following relation

$$
\int_{t=0}^{x} e^{-t \mathbf{Z}} d t \mathbf{Z}=\mathbf{I}-e^{-x \mathbf{Z}}
$$

which leads to

$$
\begin{aligned}
& E\left[\int_{t=d_{k}}^{d_{k+1}} e^{-X(t) v} \mathbf{1}_{(\Omega(t))} d t\right]((\mathbf{R}-d \mathbf{I}) v-\mathbf{Q})= \\
& \int_{x_{0}} \mathbf{f}\left(x_{0}\right) \int_{x_{1}} \mathbf{A}\left(\frac{x_{0}}{d}, x_{1}\right) \ldots \int_{x_{k}} \mathbf{A}\left(\frac{x_{k-1}}{d}, x_{k}\right)\left(\mathbf{I}-e^{\left.-\frac{x_{k}}{d}(\mathbf{R}-d \mathbf{I}) v-\mathbf{Q}\right)}\right) e^{-x_{k} v} d x_{k} \ldots d x_{0}= \\
& \int_{x_{0}} \mathbf{f}\left(x_{0}\right) \int_{x_{1}} \mathbf{A}\left(\frac{x_{0}}{d}, x_{1}\right) \ldots \int_{x_{k-1}} \mathbf{A}\left(\frac{x_{k-2}}{d}, x_{k-1}\right) \\
& \left(\int_{x_{k}} \mathbf{A}\left(\frac{x_{k-1}}{d}, x_{k}\right) e^{-x_{k} v} d x_{k}-\int_{x_{k}} \mathbf{A}\left(\frac{x_{k-1}}{d}, x_{k}\right) e^{-\frac{x_{k}}{d}(\mathbf{R} v-\mathbf{Q})} d x_{k}\right) d x_{k-1} \ldots d x_{0}= \\
& \int_{x_{0}} \mathbf{f}\left(x_{0}\right) \int_{x_{1}} \mathbf{A}\left(\frac{x_{0}}{d}, x_{1}\right) \ldots \int_{x_{k-1}} \mathbf{A}\left(\frac{x_{k-2}}{d}, x_{k-1}\right) \\
& \left(\mathbf{A}^{*}\left(\frac{x_{k-1}}{d}, v\right)-\mathbf{A}^{*}\left(\frac{x_{k-1}}{d}, \mathcal{O}(v)\right)\right) d x_{k-1} \ldots d x_{0}= \\
& \int_{x_{0}} \mathbf{f}\left(x_{0}\right) \int_{x_{1}} \mathbf{A}\left(\frac{x_{0}}{d}, x_{1}\right) \ldots \int_{x_{k-1}} \mathbf{A}\left(\frac{x_{k-2}}{d}, x_{k-1}\right) \\
& \left(e^{-x_{k-1} \mathcal{O}(v)}-e^{-x_{k-1} \mathcal{O}^{2}(v)}\right) d x_{k-1} \ldots d x_{0}= \\
& \int_{x_{0}} \mathbf{f}\left(x_{0}\right) \int_{x_{1}} \mathbf{A}\left(\frac{x_{0}}{d}, x_{1}\right) \ldots \int_{x_{k-2}} \mathbf{A}\left(\frac{x_{k-3}}{d}, x_{k-2}\right) \\
& \left(\mathbf{A}^{*}\left(\frac{x_{k-2}}{d}, \mathcal{O}(v)\right)-\mathbf{A}^{*}\left(\frac{x_{k-2}}{d}, \mathcal{O}^{2}(v)\right)\right) d x_{k-2} \ldots d x_{0}= \\
& \ldots=\int_{x_{0}} \mathbf{f}\left(x_{0}\right)\left(\mathbf{A}^{*}\left(\frac{x_{0}}{d}, \mathcal{O}^{k-1}(v)\right)-\mathbf{A}^{*}\left(\frac{x_{0}}{d}, \mathcal{O}^{k}(v)\right)\right) d x_{0}= \\
& \mathbf{f}^{*}\left(\mathcal{O}^{k}(v)\right)-\mathbf{f}^{*}\left(\mathcal{O}^{k+1}(v)\right)
\end{aligned}
$$

which completes the proof of the proposition. 
Theorem 4. In the stable fluid vacation model with exhaustive discipline the following relation holds for the steady-state vector LT of the fluid level at arbitrary epoch

$$
\mathbf{q}^{*}(v)(\mathbf{R} v-\mathbf{Q})((\mathbf{R}-d \mathbf{I}) v-\mathbf{Q})=\frac{v d}{c}\left(\mathbf{f}^{*}(v)-\mathbf{m}\right) .
$$

Proof. The fluid level at arbitrary epoch can be expressed by the help of the fluid level at the last service start on LT level by utilizing the transient behavior of the arrived fluid (relation (7)) and taking into account that it can fall either in service or vacation period as well as its position in the actual period. Thus it is enough to average over a cycle for determining the behavior at arbitrary epoch.

$$
\begin{aligned}
\mathbf{q}^{*}(v) & =\frac{1}{c} E\left[\int_{t=0}^{C} e^{-X(t) v} \mathbf{1}_{(\Omega(t))} d t\right] \\
& =\frac{1}{c}\left(\sum_{k=0}^{\infty} E\left[\int_{t=d_{k}}^{d_{k+1}} e^{-X(t) v} \mathbf{1}_{(\Omega(t))} d t\right]+E\left[\int_{t=S}^{C} e^{-X(t) v} \mathbf{1}_{(\Omega(t))} d t\right]\right)
\end{aligned}
$$

From Proposition 5 we have

$$
\begin{aligned}
& E\left[\int_{t=0}^{S} e^{-X(t) v} \mathbf{1}_{(\Omega(t))} d t\right]((\mathbf{R}-d \mathbf{I}) v-\mathbf{Q})= \\
& \sum_{k=0}^{\infty} E\left[\int_{t=d_{k}}^{d_{k+1}} e^{-X(t) v} \mathbf{1}_{(\Omega(t))} d t\right]((\mathbf{R}-d \mathbf{I}) v-\mathbf{Q})= \\
& \sum_{k=0}^{\infty} \mathbf{f}^{*}\left(\mathcal{O}^{k}(v)\right)-\mathbf{f}^{*}\left(\mathcal{O}^{k+1}(v)\right)=\mathbf{f}^{*}(v)-\mathbf{f}^{*}\left(\mathcal{O}^{\infty}(v)\right)=\mathbf{f}^{*}(v)-\mathbf{m}
\end{aligned}
$$

where we used the stationary version of (18) in the last step. For the evaluation of $E\left[\int_{t=S}^{C} e^{-X(t) v} \mathbf{1}_{(\Omega(t))} d t\right]$ it is enough to originate the fluid level from the start of the vacation instead of the last service start. This is because (34) expresses $\mathbf{q}^{*}(v)$ as sum of expected values. Relying on this and using again (32) we get for the vacation period

$$
\begin{aligned}
& E\left[\int_{t=S}^{C} e^{-X(t) v} \mathbf{1}_{(\Omega(t))} d t\right](\mathbf{R} v-\mathbf{Q})=\int_{t=0}^{\infty} \int_{x=0}^{t} \mathbf{m A}^{*}(x, v) d x \sigma(t) d t(\mathbf{R} v-\mathbf{Q})= \\
& \mathbf{m} \int_{t=0}^{\infty} \int_{x=0}^{t} e^{-x(\mathbf{R} v-\mathbf{Q})} d x \sigma(t) d t(\mathbf{R} v-\mathbf{Q})=\mathbf{m} \int_{t=0}^{\infty}\left(\mathbf{I}-e^{-t(\mathbf{R} v-\mathbf{Q})}\right) \sigma(t) d t= \\
& \mathbf{m}-\mathbf{m} \sigma^{*}(\mathbf{R} v-\mathbf{Q})=\mathbf{m}-\mathbf{f}^{*}(v),
\end{aligned}
$$

where we used (23) in the last step.

Multiplying both sides of (34) by $(\mathbf{R} v-\mathbf{Q})((\mathbf{R}-d \mathbf{I}) v-\mathbf{Q})$ and substituting (35) and (36) we get statement of the theorem. 


\section{Moments of the stationary performance measures}

The goal of this section is to obtain computable moments expressions based on the transform domain expressions of the previous section.

Lemma 1. In the stable fluid vacation model with exhaustive discipline the steady-state vector mean of the fluid level at arbitrary epoch is

$$
\mathbf{q}^{(1)}=\mathbf{q}^{(1)} \mathbf{e} \boldsymbol{\pi}+\left(\boldsymbol{\pi} \mathbf{R Q}+\frac{d}{c}\left(\mathbf{f}^{(0)}-\mathbf{m}\right)\right)\left(\mathbf{Q}^{2}+\mathbf{e} \boldsymbol{\pi}\right)^{-1},
$$

where

$$
\begin{gathered}
\mathbf{q}^{(1)} \mathbf{e}=\frac{1}{\lambda}\left(\frac{1}{2} \mathbf{t}^{(2)} \mathbf{e}-\left(\boldsymbol{\pi} \mathbf{R}-\mathbf{t}^{(1)}\right)(\mathbf{Q}+\mathbf{e} \boldsymbol{\pi})^{-1} \mathbf{R e}\right), \\
\mathbf{t}^{(1)} \mathbf{e}=\frac{1}{\lambda-d}\left(\frac{1}{2} \mathbf{r}^{(2)} \mathbf{e}+\mathbf{r}^{(1)}(\mathbf{Q}+\mathbf{e} \boldsymbol{\pi})^{-1}(\mathbf{R}-d \mathbf{I}) \mathbf{e}\right), \\
\mathbf{t}^{(1)}=\mathbf{t}^{(1)} \mathbf{e} \boldsymbol{\pi}-\mathbf{r}^{(1)}(\mathbf{Q}+\mathbf{e} \boldsymbol{\pi})^{-1}, \\
\mathbf{t}^{(2)} \mathbf{e}=\frac{1}{\lambda-d}\left(\frac{1}{3} \mathbf{r}^{(3)} \mathbf{e}-\left(2 \mathbf{t}^{(1)}-\mathbf{r}^{(2)}\right)(\mathbf{Q}+\mathbf{e} \boldsymbol{\pi})^{-1}(\mathbf{R}-d \mathbf{I}) \mathbf{e}\right),
\end{gathered}
$$

and

$$
\mathbf{r}^{(1)}=\frac{d}{c} \mathbf{m}\left(\sigma^{*}(-\mathbf{Q})-\mathbf{I}\right), \quad \mathbf{r}^{(n)}=\frac{n d}{c} \mathbf{m} \sigma^{(n-1)} \quad \forall n \geq 1 .
$$

Proof. The derivative of $(33)$ at $v=0$ gives

$$
\mathbf{q}^{(1)} \mathbf{Q}^{2}-\mathbf{q}^{(0)} \mathbf{R Q}-\mathbf{q}^{(0)} \mathbf{Q}(\mathbf{R}-d \mathbf{I})=\frac{d}{c}\left(\mathbf{f}^{(0)}-\mathbf{m}\right) .
$$

Since $(\mathbf{Q}+\mathbf{e} \boldsymbol{\pi})$ as well as $\left(\mathbf{Q}^{2}+\mathbf{e} \boldsymbol{\pi}\right)$ are nonsingular $[4,5]$, using $\mathbf{q}^{(0)}=\boldsymbol{\pi}$, $\boldsymbol{\pi} \mathbf{Q}=\mathbf{0}, \boldsymbol{\pi}\left(\mathbf{Q}^{2}+\mathbf{e} \boldsymbol{\pi}\right)^{-1}=\boldsymbol{\pi}$ and adding and subtracting $\mathbf{q}^{(1)} \mathbf{e} \boldsymbol{\pi}$ we obtain (37), where the only remaining unknown is the scalar $\mathbf{q}^{(1)} \mathbf{e}$, because according to $(23) \mathbf{f}^{(0)}=\mathbf{m} \sigma^{*}(-\mathbf{Q})$.

Unfortunately the computation of $\mathbf{q}^{(1)} \mathbf{e}$ is not that straight forward. To derive it we adopt a two step method. According to the structure of (33) we introduce $\mathbf{t}^{*}(v)=\mathbf{q}^{*}(v)(\mathbf{R} v-\mathbf{Q})$ and $\mathbf{r}^{*}(v)=\mathbf{t}^{*}(v)((\mathbf{R}-d \mathbf{I}) v-\mathbf{Q})$ and express the moments of $\mathbf{q}^{*}(v)$ by the moment of $\mathbf{t}^{*}(v)$ in the first step and express the moments of $\mathbf{t}^{*}(v)$ by the moment of $\mathbf{r}^{*}(v)$ in the second step. Considering $\mathbf{q}^{(0)}=$ $\boldsymbol{\pi}$, the first two derivatives of $\mathbf{t}^{*}(v)=\mathbf{q}^{*}(v)(\mathbf{R} v-\mathbf{Q})$ at $v=0$ are

$$
\begin{gathered}
\mathbf{t}^{(1)}=-\mathbf{q}^{(1)} \mathbf{Q}+\boldsymbol{\pi} \mathbf{R}, \\
\mathbf{t}^{(2)}=-\mathbf{q}^{(2)} \mathbf{Q}+2 \mathbf{q}^{(1)} \mathbf{R} .
\end{gathered}
$$

Adding and subtracting $\mathbf{q}^{(1)} \mathbf{e} \boldsymbol{\pi}$ to (40) and using $\boldsymbol{\pi}(\mathbf{Q}+\mathbf{e} \boldsymbol{\pi})^{-1}=\boldsymbol{\pi}$ leads to

$$
\mathbf{q}^{(1)}=\left(\mathbf{q}^{(1)} \mathbf{e}\right) \boldsymbol{\pi}+\left(\boldsymbol{\pi} \mathbf{R}-\mathbf{t}^{(1)}\right)(\mathbf{Q}+\mathbf{e} \boldsymbol{\pi})^{-1} .
$$


Post-multiplying (41) by e, post-multiplying (42) by $\mathbf{R e}$ using $\boldsymbol{\pi} \mathbf{R e}=\lambda$ and substituting the $\mathbf{q}^{(1)} \mathbf{R e}$ term from one equation to the other we obtain (38), where the unknowns are $\mathbf{t}^{(2)} \mathbf{e}$ and $\mathbf{t}^{(1)}$. Similarly, the $n$th derivative of $\mathbf{r}^{*}(v)=$ $\mathbf{t}^{*}(v)((\mathbf{R}-d \mathbf{I}) v-\mathbf{Q})$ at $v=0$ is

$$
\mathbf{r}^{(n)}=-\mathbf{t}^{(n)} \mathbf{Q}+n \mathbf{t}^{(n-1)}(\mathbf{R}-d \mathbf{I}) .
$$

Applying the same steps as in the transformation of (40) to (42) we obtain

$$
\mathbf{t}^{(n)}=\mathbf{t}^{(n)} \mathbf{e} \boldsymbol{\pi}+\left(n \mathbf{t}^{(n-1)}(\mathbf{R}-d \mathbf{I})-\mathbf{r}^{(n)}\right)(\mathbf{Q}+\mathbf{e} \boldsymbol{\pi})^{-1},
$$

and applying the same steps as in the derivation of (38) based on (41) and (42) we obtain

$$
\mathbf{t}^{(n)} \mathbf{e}=\frac{1}{\lambda-d}\left(\frac{1}{n+1} \mathbf{r}^{(n+1)} \mathbf{e}-\left(n \mathbf{t}^{(n-1)}-\mathbf{r}^{(n)}\right)(\mathbf{Q}+\mathbf{e} \boldsymbol{\pi})^{-1}(\mathbf{R}-d \mathbf{I}) \mathbf{e}\right)
$$

Considering that $\mathbf{t}^{(0)}=-\mathbf{q}^{(0)} \mathbf{Q}=\mathbf{0}$, (44) and (45) allows the consecutive computation of $\mathbf{t}^{(n)} \mathbf{e}$ and $\mathbf{t}^{(n)}$. Finally, using (23) the derivatives of $\mathbf{r}^{*}(v)=$ $\frac{v d}{c}\left(\mathbf{f}^{*}(v)-\mathbf{m}\right)$ at $v=0$ gives $(39)$.

\section{Conclusion}

Finally, the steady-state mean vector $\mathbf{q}^{(1)}$ can be computed by the following steps.

1. Calculation of the steady-state phase distribution of the background Markov chain at start of vacation, $\mathbf{m}$, form the system of linear equations (22).

2. Computation of $\boldsymbol{\pi}, \lambda, \rho$ and $c$ by applying (1), (2) and (27), respectively.

3. Computation of the steady-state mean $\mathbf{q}^{(1)}$ by applying Lemma 1.

\section{Acknowledgment}

This work is supported by the OTKA K101150 project.

\section{References}

1. S. C. Borst and O. J. Boxma. Polling models with and without switchover times. Operations Research, 45:536-543, 1997.

2. B. T. Doshi. Queueing systems with vacations - a survey. Queueing Systems, 1:29 66, 1986.

3. S. Gerschgorin. Über die Abgrenzung der Eigenwerte einer Matrix. Izv. Akad. Nauk. USSR Otd. Fiz.-Mat. Nauk, 7:749-754, 1931.

4. D. L. Lucantoni. New results on the single server queue with a batch markovian arrival process. Stochastic Models, 7:1-46, 1991.

5. M. F. Neuts. Structured stochastic matrices of $M / G / 1$ type and their applications. Marcel Dekker, New York, 1989.

6. Z. Saffer and M. Telek. Fluid vacation model with markov modulated load and gated discipline. In QTNA, Aug. 2014.

7. H. Takagi. Queueing Analysis - A Foundation of Performance Evaluation, Vacation and Prority Systems, vol.1. North-Holland, New York, 1991. 